\title{
Some Simple Mixing and Mass Matrices for Neutrinos
}

\author{
Xiao-Gang $\mathrm{He}^{a}$ and A. Zee ${ }^{b}$ \\ a Department of Physics, National Taiwan University, Taipei, Taiwan \\ ${ }^{b}$ Institute for Theoretical Physics, University of California, Santa Barbara, California 93106, \\ $U S A$
}

\begin{abstract}
We argue that the accumulated neutrino data, including recent results from KamLAND and K2K, point to a neutrino mixing matrix with $\left(V_{11}, V_{21}, V_{31}\right.$; $\left.V_{21}, V_{22}, V_{32} ; V_{13}, V_{23}, V_{33}\right)=(-2 / \sqrt{6}, 1 / \sqrt{6}, 1 / \sqrt{6} ; 1 / \sqrt{3}, 1 / \sqrt{3}, 1 / \sqrt{3} ;$

$0,1 / \sqrt{2},-1 / \sqrt{2})$. We propose some simple neutrino mass matrices which predict such a mixing matrix.
\end{abstract}

In this brief note, we suggest that the accumulating neutrino data [1-3], including the recent results from KamLAND [4] and K2K [5], point to a relatively simple neutrino mass matrix. The data can be explained by oscillations between three active neutrinos with the atmospheric neutrino and $\mathrm{K} 2 \mathrm{~K}$ data explained by oscillation between the muon and the tauon neutrinos, and the solar neutrino and KamLAND data explained by the oscillation between the electron and muon neutrinos. Following standard convention, let us denote the neutrinos current eigenstates, coupled to the charged leptons by the $W$ bosons, by $\nu_{\alpha}$ $(\alpha=e, \mu, \tau)$ and the neutrino mass eigenstates by $\nu_{i}(i=1,2,3)$. We will take the neutrinos to be Majorana as seems likely [6], and thus the neutrino mass matrix $M_{\alpha \beta}$ is symmetric in the basis of the current eigenstates. We will also for simplicity assume CP conservation so that $M$ is real. Thus, $M$ is diagonalized by an orthogonal transformation

$$
V^{T} M V=D
$$


where the diagonal matrix $D$ has entries $m_{1}, m_{2}$, and $m_{3}$. Clearly, we are free to multiply the Maki-Nakagawa-Sakata [7] mixing matrix $\mathrm{V}$ on the right by some diagonal matrix whose diagonal entries are equal to \pm 1 . This merely multiplies each of the columns in $\mathrm{V}$ by an arbitrary sign.

We refer the reader to the literature for a detailed analysis of the data [8-10]. For our purposes, the data may be summarized as follows. Define the mass squared difference by $\Delta m_{i j}^{2} \equiv m_{i}^{2}-m_{j}^{2}$. At the $99.3 \%$ confidence level $\Delta m_{i j}^{2}$ are constrained by

$$
1.5 \times 10^{-3} \mathrm{eV}^{2} \leq\left|\Delta m_{32}^{2}\right| \leq 5.0 \times 10^{-3} \mathrm{eV}^{2}
$$

and

$$
2.2 \times 10^{-5} e V^{2} \leq\left|\Delta m_{21}^{2}\right| \leq 2.0 \times 10^{-4} e V^{2}
$$

with the best fit values given by $\left|\Delta m_{32}^{2}\right|=3.0 \times 10^{-3} \mathrm{eV}^{2}$ and $\left|\Delta m_{21}^{2}\right|=7.0 \times 10^{-5} \mathrm{eV}$. The mixing angles are in the ranges of $\sin ^{2} 2 \theta_{23}>0.85$ and $0.18 \leq \sin ^{2} \theta_{12} \leq 0.37$. Finally, the CHOOZ experiment, done with a French reactor, failed to see the disappearance of electron antineutrino and thus gives an upper bound of about 0.22 on the $\nu_{e}-\nu_{\tau}$ oscillation parameter $\left|V_{13}\right|[3]$.

We interpret the data on the mixing angles as follows. The CHOOZ experiment indicates that $V_{13}$ is small and so we will simply set it to 0 . We choose, within the allowed experimental range, $V_{12} \simeq \sin \theta_{12} \sim 1 / \sqrt{3}$ so that $\tan ^{2} \theta_{12} \sim 1 / 2$. This is well within the range $0.37 \leq$ $\tan ^{2} \theta_{12} \leq 0.60$ at the $1 \sigma$ level indicated by the recent analysis ${ }^{1}$. Finally, the atmospheric neutrino data [2] and $\mathrm{K} 2 \mathrm{~K}$ data [4] requires $V_{23} \sim 1 / \sqrt{2}$. In other words, we propose that we know the upper triangle entries of the matrix $V$ :

\footnotetext{
${ }^{1}$ See for example eq. (3.1) of Bahcall et al. in Ref. [10]. The bi-maximal matrix gives a too large $\tan \theta_{12}=1[11]$
} 


$$
V=\left(\begin{array}{ccc}
X & \frac{1}{\sqrt{3}} & 0 \\
X & X & \frac{1}{\sqrt{2}} \\
X & X & X
\end{array}\right),
$$

where $X$ denotes an unknown quantity.

Remarkably, this essentially fixes the mixing matrix $V$. Once we take the last column to be proportional to $(0,1,-1)$, orthogonality and our " knowledge" that $\left|V_{12}\right|$ is $1 / \sqrt{3}$ immediately fix the second column to be proportional $(1,1,1)$ and hence the first column to be proportional to $(-2,1,1)$. We therefore obtain ${ }^{2}$,

$$
V=\left(\begin{array}{ccc}
-\frac{2}{\sqrt{6}} & \frac{1}{\sqrt{3}} & 0 \\
\frac{1}{\sqrt{6}} & \frac{1}{\sqrt{3}} & \frac{1}{\sqrt{2}} \\
\frac{1}{\sqrt{6}} & \frac{1}{\sqrt{3}} & -\frac{1}{\sqrt{2}}
\end{array}\right) .
$$

As remarked earlier, we are free to choose the signs of the column vectors in the mixing matrix and to make chiral rotations on the neutrino fields to change the relative signs of the mass eigenvalues ${ }^{3}$.

The three column vectors contained in $V$ are the eigenvectors of the matrix

$$
M_{0}=a\left(\begin{array}{rrr}
2 & 0 & 0 \\
0 & -1 & 3 \\
0 & 3 & -1
\end{array}\right) \text {, }
$$

with eigenvalues $m_{1}=m_{2}=2 a$, and $m_{3}=-4 a$. (The parameter $a$ merely sets the overall scale.) Thus, $\Delta m_{21}^{2}=0$ and this pattern reproduces the data $\left|\Delta m_{21}^{2}\right| /\left|\Delta m_{32}^{2}\right| \ll 1$ to first

\footnotetext{
${ }^{2}$ Thus $\mathrm{V}$ has the pleasing form that its three columns just correspond to the three diagonal Gell-Mann matrices of $\mathrm{U}(3)$.

${ }^{3}$ Without information on the relative signs of the eigen-masses, the column vectors can only be determined up to $\pm i$. This can be expressed by multiplying a diagonal phase matrix $P=$ $\operatorname{Diag}\left(e^{i \sigma}, e^{i \rho}, 1\right)$ to the right of $\mathrm{V}$. With $\mathrm{CP}$ invariance, $\sigma$ and $\rho$ can take the values of zero or $\pm \pi / 2$. Neutrinoless double beta decays will provide some crucial information on these phases.
} 
approximation. Because of the degeneracy in the eigenvalue spectrum, $V$ is not uniquely determined. We can always replace $V$ by $V W$ where

$$
W=\left(\begin{array}{ll}
R & 0 \\
0 & 1
\end{array}\right),
$$

with $R$ a $2 \times 2$ rotation matrix. To determine $V$, and at the same time to split the degeneracy between $m_{1}$ and $m_{2}$, we perturb $M_{0}$ to $M=M_{0}+\delta M_{T}$, where

$$
\delta M_{T}=\varepsilon a\left(\begin{array}{ccc}
0 & 1 & 1 \\
1 & 0 & 1 \\
1 & 1 & 0
\end{array}\right) .
$$

We have the mass eigenvalues $m_{1}=2 a(1-\varepsilon / 2), m_{2}=2 a(1+\varepsilon)$, and $m_{3}=-4 a(1+\varepsilon / 4)$. Thus, to the lowest order, we can determine $\varepsilon=\Delta m_{21}^{2} / \Delta m_{32}^{2}$. The overall scale of the mass matrix $a$ is given by $a^{2}=\Delta m_{32}^{2} / 12$.

Note that our proposed neutrino Majorana mass matrix $M$ is traceless. One may be tempted to conjecture that this property may provide a clue to the origin of the mass matrix M. As is well known, a general Majorana matrix for the neutrinos has 9 real parameters while feasible experiments can measure only 7 of these. It has been suggested that conditions such as $\operatorname{Det} M=0$ [12], texture zeros or other relations [13-17] be imposed to cut down on the number of parameters. Our example here satisfies $\operatorname{Tr} M=0$, but not $\operatorname{Det} M=0$. In a forthcoming paper [18], we give a phenomenological analysis of the data imposing the condition $\operatorname{Tr} M=0$, which is generally satisfied by models in which $M$ is given by the commutator $^{4}$ of two matrices $M=[A, B]$.

Other perturbations can also lead to the same mixing matrix $V$ while splitting the degeneracy $\Delta m_{21}^{2}=0$. An interesting example is the "democratic" form

\footnotetext{
${ }^{4} \mathrm{An}$ example is the simplest version of the many so-called Zee models [12].
} 


$$
\delta M_{D}=\varepsilon a\left(\begin{array}{lll}
1 & 1 & 1 \\
1 & 1 & 1 \\
1 & 1 & 1
\end{array}\right)
$$

The matrix $\delta M_{D}$ is evidently a projection matrix that projects the first and third columns in $\mathrm{V}$ to zero. Thus, the eigenvalues are given by $m_{1}=2 a, m_{2}=2 a(1+3 \varepsilon / 2)$, and $m_{3}=-4 a$. Again $\varepsilon$ and $a^{2}$ are given by, to the lowest order, $\varepsilon=\Delta m_{21}^{2} / \Delta m_{32}^{2}$ and $a^{2}=\Delta m_{32}^{2} / 12$, respectively. We note that this mass matrix is not traceless.

We mention that there is a whole class of models we can propose. Generalize $M_{0}$ to be

$$
\tilde{M}_{0}=a\left(\begin{array}{ccc}
2 & 0 & 0 \\
0 & 1-y & 1+y \\
0 & 1+y & 1-y
\end{array}\right),
$$

with the case mentioned earlier corresponding to $y=2$. Thus in general we propose

$$
M=\tilde{M}_{0}+\delta M,
$$

with $\delta M$ being $\delta M_{T}$ or $\delta M_{D}$. They lead to the same mixing matrix $V$, with the eigenvalues $m_{i}$ given by $(2 a[1-\varepsilon / 2], 2 a[1+\varepsilon],-2 a[y+\varepsilon / 2])$ and $(2 a, 2 a[1+3 \varepsilon / 2],-2 a y)$, respectively.

Note that the most general mass matrix which produces the mixing matrix $\mathrm{V}$ can be expressed as linear combinations of the three matrices of the forms given by $M_{0}, \delta M_{T}$ and $\delta M_{D}$. Once we committed to a specific form for $M$, the three parameters specifying the linear combination merely parameterize the three neutrino eigen-masses $m_{1,2,3}$. Also for any given mixing matrix, the mass matrix can be specified by mass eigenvalues.

Our purpose here is evidently not to give a detailed fit to the data, but to suggest some relatively simple and appealing mass matrices. The appearance of simple integers in the mixing and mass matrices we proposed is perhaps intriguing and provides a glimmer of a hope that they may be obtained by group theoretic considerations. To provide a theoretical origin of the mass matrix $M$ presents an interesting challenge. 


\section{Acknowledgments}

This work was supported in part by NSC under grant number NSC 91-2112-M-002-42, and by the MOE Academic Excellence Project 89-N-FA01-1-4-3 of Taiwan, and by NSF under grant number PHY 99-07949 of USA. AZ thanks Professor Pauchy Hwang and the Department of Physics of the National Taiwan University, where this work was initiated, for warm hospitality.

\section{Note Added}

It has been called to our attention that the mixing matrix $V$ we suggested was proposed by L. Wolfenstein [19] more than 20 years ago (but with the first and second column interchanged), and by P.F. Harrison, D.H. Perkins and W.G. Scott [20] before the SNO and KamLAND data came out, and had been studied by them and by Z.-Z. Xing [21,22]. Our discussion of the mass matrix, however, seems to be novel. The mixing matrix $V$ is a special case of a family of mixing matrices obtained by C.S. Lam [23] by imposing "2-3 symmetry" . He fixed $V$ further by fitting it to the data available at the time. 


\section{REFERENCES}

[1] Q.R. Ahmad et al., (SNO Collaboration), Phys. Rev. Lett. 89, 011301(2002); Phys. Rev. Lett. 89, 011302(2002); S. Fukuda et al., (Super-Kamiokande Collaboration), Phys. Lett. B539, 179(2002); B.T. Cleveland et al., Astrophys. J. 496, 505(1998); R. Davis, Prog. Part. Nucl. Nucl. Phys. 32, 13(1994); D. N. Abdurashitov et al., (SAGE Collaboration), Phys. Rev. D60, 055801(1999); arXiv: astro-ph/0204245; W. Hampel et al., (GALLEX Collaboration), Phys. Let. B447, 127(1999); C. Cattadori, (GNO Collaboration), Nucl. Phys. B111 (Proc. Suppl.), 311(2002).

[2] Y. Fukuda et al., (Kamiokande Collaboration), Phys. Lett. B335, 237(1994); R. Becker-Szendy et al., (IMB Collaboration), Nucl. Phys. B38 (Proc. Suppl.), 331(1995); W.W.M. Allison et al., (Soudan Collaboration) Phys. Lett. B449, 137(1999); M. Ambrosio et al., (MACRO Collaboration) Phys. Lett. B434, 451(1998); M. Soili, arXiv: hep-ex/0211030.

[3] M. Apollonio et al., (CHOOZ Collaboration), Phys. Lett. 466, 415(1999) [arXiv: hep$\mathrm{ph} / 9907037]$.

[4] K. Eguchi et al., (KamLAND Collaboration), arXiv:hep-ph/0212021.

[5] M.H. Ahn et al., (K2K Collaboration), arXiv:hep-ph/0212007.

[6] For a review, see for example, B. Kayser, arXiv: hep-ph/0211134.

[7] Z. Maki, M. Nakagawa and S. Sakata, Prog. Theor. Phys. 28, 870(1962).

[8] V. Barger et al., Phys. Lett. B537,179(2002) [arXiv: hep-ph/0204253]; J.N. Bachall, M.C. Gonzalez-Garcia and C. Pena-Garay, JHEP 0207, 054(2002) [arXiv: hepph0204314]; M. Maltoni et al., arXiv: hep-ph/0207227; G.L. Fogli et al., arXiv: hepph/0208026.

[9] P. Creminelli, G. Signorelli and A. Strumia, JHEP 0105:052(2001) (Addendum) [arXiv: hep-ph/0102234]; V. Barger and D. Marfatia, arXiv: hep-ph/0212126; G. Fogli et al., 
arXiv: hep-ph/0212127; M. Maltoni, T. Schwetz and J. Valle, arXiv: hep-ph/0212129; A. Bandyopadhyay et al., arXiv: hep-ph/0212146; H. Nunokawa and W. Teves and R. Z. Funchal, hep-ph/0212202; P. Aliani et al., arXiv: hep-ph0212212; P.C. de Holanda and A. Yu. Smirnov, arXiv hep-ph/0212270; S. Pakvasa and J. Valle, arXiv: hepph/0301061; A. Balantekin and H. Yuksel, arXiv: hep-ph/0301072.

[10] J.N. Bachall, M.C. Gonzalez-Garcia and C. Pena-Garay, arXiv: hep-ph/0212147.

[11] V. Barger, S. Pakvasa, T. Weiler and K. Whisnant, Phys. Lett. B437, 107(1998).

[12] G.C. Branco, R. Felipe, F. Joaquim and T. Yanagida, arXiv: hep-ph/0212341.

[13] A. Zee, Phys. Lett. 93B, 389(1980); 161B, 141(1985); L. Wolfenstein, Nucl. Phys. B175, 93(1980); For a brief review, see D. Chang and A. Zee, Phys. Rev. D61, 071303(2000).

[14] Y. Smirnov and Zhijian Tao, Nucl. Phys. B426, 415(1994); Y. Smirnov and M. Tanimoto, Phys. Rev. D55, 1665(1997); C. Jarlskog et al., Phys. Lett. B449, 240(1999); P. Frampton and S. Glashow, Phys. Lett. B461, 95(1999).

[15] Y. Koide, Phys. Rev. D64, 077301(2001); P. Frampton, M. Oh and T. Yoshikawa, Phys. Rev. D65, 073014(2002).

[16] A. Joshipura and S. Rindani, Phys. Lett. B464, 239(1999); K.-M. Cheung and O. Kong, Phys. Rev. D16, 113012(2000); K. Baliji, W. Grimus and T. Schwetz, Phys. Lett. B508, 301(2001); E. Mitsuda and K. Sasaki, Phys. Lett. B516, 47(2001); A. Ghosal, Y. Koide and H. Fusaoka, Phys. Rev. D64, 053012(2001); D. Dicus, H.-J. He and J. Ng, Phys. Rev. Lett. 87, 111803(2001); B. Brahmachari and S. Choubey, Phys. Lett. B531, 99(2002); T. Kitabayashi and M. Yasue, Int.J. Mod. Phys. A17, 2519(2002); M.Y. Cheng and K. Cheung, arXiv: hep-ph/0203051; Y. Koide, Nucl. Phys. Proc. Suppl. 111, 294(2002).

[17] P. H. Frampton, S.L. Glashow and D. Marfatia, Phys. Lett. B 536, 79(2002) [arXiv: 
hep-ph/0202008]; M.-C. Chen and K.T. Mahanthappa, arXiv: hep-ph/0212375; B. Desai, D. Roy and A, Vaucher, arXiv: hep-ph/02035; W.-L. Guo and Z.-Z. Xing, arXiv: hep-ph/0212142; Z.-Z. Xing, arXiv: hep-ph/0210276; E. Ma, Phys. Rev. D66, 117301(2002); A. Kageyama et al., Phys. Rev. D65, 096010(2002); S. Barr, J. Int. Mod. Phys. A16S1B, 579(2001); I. Dorsner and S. Barr, Nucl. Phys. B617, 493(2001); A.J. Davies and X.-G. He, Phys. Rev. D46, 3208(1992); K. Babu and Q. Shfi, Phys. Lett. B311, 172(1993); J. H. Hewett and T.G. Rizzo, Phys. Rev. D33, 1519(1996).

[18] X.-G. He and A. Zee, in preparation.

[19] L. Wolfenstein, Phys. Rev. D18, 958(1978).

[20] P.F. Harrison, D. H. Perkins and W.G. Scott, Phys. Lett. B458, 79(1999).

[21] W.G. Scott, Nucl. Phys. Proc. Suppl. 85, 177(2000) [arXiv: hep-ph/9909431]; P. F. Harrison, D.H. Perkins and W.G. Scott, Phys. Lett. B530, 167(2002) [arXiv: hepph/0202074]; P. F. Harrison and W.G. Scott, Phys. Lett. B535, 163(2002); arXiv: hep-ph/0302025.

[22] Z.-Z. Xing, Phys. Lett. B533, 85(2002) [arXiv: hep-ph/0204049].

[23] C.S. Lam Phys. Lett. B507, 214(2002) [arXiv: hep-ph/0104116]. 